\title{
La présence de Bogidiella albertimagni Hertzog 1933 en Roumanie et quelques remarques sur les espèces européennes du genre
}

\author{
Par Dan. Dancau et Eugen Serban ${ }^{1}$ )
}

Avec planches et 1 figure dans le texte $\% 1(1)-75$ (5)

A l'occasion de récentes recherches sur la faune phréatique de la Vallée de la Cerna (région Olténie), nous avons retrouvé le genre Bogidiella, signalé pour la première fois en Roumanie par E. Dobreanu et C. Manolache (1951 - Bogidiella skopljensis Karaman), d'après le matériel procuré par M. le Prof. Tr. Orghidan.

Les individus capturés appartiennent à l'espèce $B$ albertimagni décrite de Strasbourg par Hertzog et retrouvée, 23 années plus tard, par Husmann (1956) dans la nappe phréatique des rivières Weser et Leine. Notre matériel a été collecté en employant le procédé des sondages Karaman-Chappuis ${ }^{2}$ ). Le seul exemplaire de 1961, assez mal conservé, ne nous a pas permis l'identification de l'espèce. Néanmoins les pléopodes à une seule branche, nous ont donné la certitude qu'il ne s'agissait pas de B.skopljensis Karaman. L'année suivante, en reprenant les recherches sur la faune de la nappe phréatique ${ }^{3}$ ) de la rivière Cerna, les 80 sondages exécutés dans le secteur Lunca LargaGura Craiovei $(10 \mathrm{~km})$, à environ $50 \mathrm{~km}$ de Baile Herculane, sont restés malheureusement sans résultats. Ce fut en 1963, au mois d'octobre (dans la période des basses eaux) que nous avons eu la chance de capturer 15 individus, dans la Vallée de Cernişoara et dans celle de la Cerna, à Cerna-sat (température: $9^{\circ} \mathrm{C}$ ).

Nous pensons qu'il est inutile de donner une description détaillée de notre matériel, pour la raison que nos exemplaires ne se distinguent pas de la description de Hertzog pour le type de Strasbourg (1936). Nous donnons, toutefois, ci-dessous une présentation sommaire à laquelle nous joignons plusieurs dessins et une photo.

1) Institut de Spéologie "E. G. Racovitza» Bucarest, Roumanie.

2) G. Motaş, Acta Muz. Maced. Sci. Natur., VIII, 7/75/, 1962.

3) Biotope dénommé par Tr. Orghidan le biotope hyporhéique, Bul. şt. Acad. R. P. R., VII, 13, 1955. 
Corps. Long de 1,7 à 2,3 mm.

Antennes I (planche $2 \mathrm{~A}$ et $4 \mathrm{~A}, \mathrm{~B}$ ) à flagelle principal de 7 articles. Le flagelle accessoire constitué de 3 articles, ne dépasse pas la longueur des 3 articles basilaires réunis du flagelle principal.

Antennes I I (planche 2B) un peu plus courtes que la paire antérieure; flagelle de 5 articles d'une longueur un peu plus réduite que celle des deux articles distaux réunis du pédoncule.

Labium (planche $4 \mathrm{C}$ ) à lobes latéraux visiblement éloignés, les médians bien individualisés et à bords apicaux arrondis.

Mandibules (planche $2 \mathrm{C}, \mathrm{D})$. Pars incisiva à 5 dents bien chitinisées; lame accessoire à 5-6 dents. Pars molaris, située à la limite inférieure du tiers distal du corps de la mandibule, est munie apicalement, d'une rosette constituée de petites dents, et d'une petite soie. Entre pars molaris et pars incisiva, plusieurs lamelles et soies (planche 2 D). Palpe robuste, à 3 articles.

Maxilles I (planche $2 \mathrm{E}$ ). Lobe externe à 7 styles, 5 ordinaires et 2 bifurqués distalement. Lobe interne avec 2 à 3 soies sur le bord distal. Le palpe, biarticulé, présente l'article proximal de 1/2 plus court que l'article distal.

Maxilles I I (planche $2 \mathrm{~F}$ ). Lobe externe à 8-9 soies, lobe interne à 5 .

Maxillipèdes (planche $2 \mathrm{G}$ et $4 \mathrm{D}$ ) pareilles, ainsi que les autres pièces, à celles des individus de Strasbourg. On peut préciser que sur l'article III du palpe - à son angle distal et interne - il existe une proéminence à 4 dents (planche $4 \mathrm{D}$ ).

Gnathopodes I et I I (planche $3 \mathrm{~A}, \mathrm{~B}$ et $4 \mathrm{E}, \mathrm{F}$ ). Gnathopodes I plus robustes que les seconds qui sont plus longs. Le bord palmaire fortement denté, par rapport au bord sternal de tous les articles, qui est finement crénelé. La région distale du bord sternal du basis porte un seul poil; le médian manque chez tous les individus. Le rapport entre la longueur du bord palmaire et celle du bord sternal du propode est égal à $1 / 2$.

Péréi o p o des I I I - V I I (planche $3 \mathrm{C}-\mathrm{G}$ ). Les deux premières paires, subégales. Les péréiopodes V, VI et VII s'allongent progressivement, les derniers étant 2 fois plus longs que la paire III. L'organe élliptique présent sur le basis de tous les péréiopodes, ayant une forme ovale (paires III-VI) ou ronde (VII). Le propode du péréiopode VII a sur son bord sternal 5 longues soies et 2 épines bifurquées distalement. Les péréiopodes VI et VII ont des dactyles fortement développés; le rapport entre la longueur de leur basis et celle de leur griffes, égal à $1 / 7$ respectivement $1 / 11$. Pour les autres paires le rapport est égal à $1 / 4-1 / 5$. 
Les branchies - 3 paires - s'insèrent sur les plaques coxales qui portent les péréiopodes IV, V, et VI.

Plaques coxales (planche 5:1-7) s'élargissent en partant de la Ire vers la VIIe. Les 4 premières ont 2 soies sur leur bord sternal; la $\mathrm{Ve}$, sur la même côté, a une seule soie et dans l'angle postérieur et tergal une autre, qui se retrouve aussi sur les plaques VI et VII.

Plaques épimérales (planche 5 D) à angle inféro-postérieur assez proéminent et arrondi.

Pléopodes (planche $5 \mathrm{~A}, \mathrm{~B}, \mathrm{C}$ ). Les pédoncules, robustes, plus longs que les branches externes, ont le retinacle constitué de 2 crochets. Branche externe à 3 articles, celle interne absente.

Uropodes (planche $5 \mathrm{E}, \mathrm{F}, \mathrm{G}$ ) à branches subégales.

Telson (planche $4 \mathrm{G}, \mathrm{H}$ et $5 \mathrm{H}$ ). Il correspond généralement à la description de Hertzog. Chez certains de nos exemplaires, il est assez semblable à celui présenté par Karaman en 1943 chez B.skopljensis (planche $5 \mathrm{H}$ ).

Depuis la découverte de Bogidiella Hertzog 1933, cet intéressant genre a été retrouvé à peu près dans tous les continents.

Ainsi donc, Ruffo (1952) décrit de l'Amérique Australe la première espèce non-européenne, B.neotropica (région des Amazones) et une année plus tard, Siewing (1953) fait connaitre, des eaux interstitielles littorales du Brésil, B.brasiliensis.

Shoemaker (1959), en étudiant des individus de Bogidiella trouvés dans la grotte «Dark Cave», Barbada (aux Antilles) signale l'unique espèce de l'Amérique Centrale, B.bredini.

Villalobos (1960) a donné la description de la seule espèce connue jusqu'à présent de l'Amérique du Nord, B.tabascensis capturée dans la grotte "Gruta del Cocona" du Mexique.

C'est toujours l'éminent spécialiste Ruffo qui signale la présence du genre dans le continent asiatique. En 1958, il décrit B.lindbergi d'Afghanistan, capturée par Lindberg dans la grotte Nayak $(2240 \mathrm{~m}$ alt.). Tout récemment (1963) le spécialiste de Verona publia une nouvelle espèce B. hebraea trouvée par F. D. Por dans une source de la dépression de la Mer Morte (-392 m alt.). De même il signale Bogidiella sp. dans l'eau souterraine littorale de l'île de la Réunion (Océan Indien).

En ce qui concerne l'Europe, on connaît jusqu'à présent 6 espèces dont une, B.albertimagni Hertzog 1933 décrite de Strasbourg, est l'espèce type du genre; quatre de Yougoslavie, B.skopljensis Karaman 1933 - décrite initialement sous le nom de Jugocrangonyx skopljensis -, B. longiflagellum Karaman 1959, B.denticulata Mestrov 1961, B. semidenticulata Mestrov 1961 et la dernière, provenant des eaux inter- 
stitielles de France et d'Algérie, B.chappuisi Ruffo 1952. Karaman a publié encore deux sous-espèces de Yougoslavie, B.albertimagni dalmatina Karaman 1953 et B.albertimagni glacialis Karaman 1959. Enfin, S. Ruffo mentionne B.denticulata Mestrov en Italie près de Verona (1963) et Bogidiella sp. en Espagne au sud de Barcelone (1953a). Spooner (1960) signale le genre dans les eaux littorales de l'Angleterre.

Comme nous l'avons déjà dit, en Roumanie le genre est représenté par Bogidiella skopljensis Karaman et B.albertimagni Hertzog. La première espèce, trouvée en Transylvanie, diffère de la population de Skoplje seulement par le flagelle accessoire de l'antenne I qui a 3 articles; la seconde est presque identique aux individus de Strasbourg selon la minutieuse description faite par Hertzog en 1936.

Malgré le fait qu'en Europe le nombre des espèces soit assez élevé, la valeur taxonomique d'une d'entre elles est assez discutable. Le matériel que nous venons de présenter étant pareil à celui de Strasbourg, nous prenons la liberté de discuter le rapport taxonomique existant entre B.albertimagni et les espèces skopljensis et denticulata de Yougoslavie.

La description très sommaire et presque simultanée de B.albertimagni et B.skopljensis ainsi que leur structure - à première vue assez peu différente -, a conduit à l'opinion que la forme de Skoplje n'est que synonyme de celle de Strasbourg: "Karamans Jugocrangonyx skopljensis 1933 - affirme Hertzog - ist, wie aus den schon veröffentlichten Daten und Figuren unzweideutig hervorgeht, mit meiner Bogidiella albertimagni 1933 identisch»(Hertzog 1935, p. 50). Cette affirmation, reprise plus tard par le même auteur, lorsqu'il donna la description détaillée de son espèce (1936), était assez justifiée à cette épogrue - là, à cause du manque de détails sur la structure de B.skopljensis. Mais, quelques années après, Karaman (1943) en publiant une description plus détaillée de l'espèce de Skoplje et en se basant spécialement sur la structure des pléopodes, arriva à la conclusion que B.skopljensis est une espèce indépendante.

En trouvant la sous-espèce B. albertimagni dalmatina de Dubrovnik, Karaman (1953) changea d'avis et, en reconsidérant la valeur taxonomique de B.skopljensis, il transforma son espèce en une sous-espèce de B.albertimagni, c'est-à-dire, B. albertimagni skopljensis $\left.{ }^{4}\right)$.

\footnotetext{
4) «Die Exemplare von Dubrovnik scheinen eine Mittelstellung zwischen skopljensis und albertimagni darzustellen. Infolgedessen können diese zwei Arten nicht mehr als getrennte Arten weiterbestehen, und skopljensis ist als Unterart zu albertimagni zu stellen, da albertimagni eine Priorität von 6 Tagen gegenüber skopljensis besitzt» (Karaman 1953, p. 142/6).
} 
La description assez succinte de cette sous-espèce ne nous permet pas d'en faire une analyse approfondie. Actuellement, on peut seulement affirmer que les caractères considérés par Karaman comme intermédiaires, ne sont en réalité que des variations individuelles chez les espèces en discussion. Par exemple, le flagelle accessoire de l'antenne I, bi- ou triarticulé est présent aussi bien chez B.albertimagni de Strasbourg $^{5}$ ), que chez B. skopljensis. L'unique exemplaire de B.skopljensis capturé en Roumanie, et qui est parfaitement semblable aux individus de Skoplje, a son flagelle triarticulé. De même, les autres caractéristiques signalées par Karaman pour B.albertimagni dalmatina ${ }^{6}$ ) sont propres à B.chappuisi Ruffo et n'appartiennent, ni à B.albertimagni, ni à B. skopljensis.

En tenant compte de ces faits, et en se basant spécialement sur la structure de leur pléopodes - uniramés chez B.albertimagni et biramés chez B.skopljensis -, S. Ruffo (1958) reste à l'opinion initiale de Karaman, en considérant la forme de Skoplje comme une véritable espèce. Il démontre, en mème temps, que B.albertimagni dalmatina est synonyme de B.chappuisi. En ce qui nous concerne, nous sommes entièrement d'accord avec Ruffo quant à la valeur taxonomique de B. skopljensis et B.albertimagni.

L'étude attentive de notre matériel nous a permis de discerner un trait qui confirme l'indépendance de ces deux espèces. Il s'agit de la structure de leurs gnathopodes I ou, plus précisément, de la position des épines marquant l'angle palmaire sur le bord sternal du propode. Chez B.skopljensis l'angle palmaire est situé à la limite distale du tiers dorsal du bord sternal, tandis que chez $B$. albertimagni il a une position médiane. Ceci détermine un bord palmaire plus court chez B.albertimagni ( $1 / 2$ du bord sternal) et un bord plus étendu chez B. skopljensis $(2 / 3$ du bord sternal). De même, cette caractéristique donne une certaine robustesse au propode du gnathopode I chez l'espèce de Strasbourg qui est moins piriforme que celui de B.skopljensis. Selon nous, la position de l'angle palmaire peut servir de critérium valable pour distinguer ces espèces, car elle est propre aussi bien aux populations de Strasbourg et d'Olténie (B.albertimagni) qu'à celles de Skoplje et de Transylvanie (B.skopljensis).

\footnotetext{
$\left.{ }^{5}\right)$ «...flagelle accessoire en général à 3 articles, parfois à 2 seulement, par coalescence des articles 1 et 2» (Hertzog 1936, p. 360).

6) «Auf den Hüften der Pereiopoden kommen auf deren Vorder- wie auch Hinterrande je einige Borsten vor" ainsi que "Das Telson scheint etwas länger als bei den anderen zwei Unterarten zu sein, es trägt auch zwei Stacheln apikal...» (Karaman 1953, p. 142/6).
} 
En ce qui concerne la synonymie $B$ albertimagni dalmatina et $B$. chappuisi, il faut préciser que récemment, Mestrov (1961), sans aucune justification, cite cette sous-espèce sous le nom de B.chappuisi dalmatina. Que ladite forme est plus rapprochée de B.chappuisi, c'est une réalité incontestable, il est assez difficile de décider sa valeur taxonomique. Si la position de Mestrov est la plus commode, cependant les caractéristiques présentées par Karaman pour cette forme, ainsi que sa présence sur le littoral de l'Adriatique, justifient pleinement l'opinion de Ruffo.

En nous rapportant à la dernière étude de ce genre en Yougoslavie Mestrov 1961 - nous constatons que B.denticulata ne présente pas les caractéristiques nécessaires pour en faire une forme nouvelle. Si les deux espèces décrites par l'auteur sus-cité, B.semidenticulata et $B$. denticulata, sont bien individualisées l'une par rapport à l'autre, en comparant la description de la dernière avec $B$.albertimagni nous n'avons pu relever des différences morphologiques.

En s'étayant sur le fait que des articles dentés chez les gnathopodes sont encore inconnus dans ce genre ${ }^{7}$ ), le spécialiste de Zagreb donne comme un trait caractéristique pour ses deux espèces les structures suivantes: "Alle Glieder des ersten und zweiten Gnathopoden außer der Palma am Propode sind am hinteren Rand sägeförmig gezähnt» (B. semidenticulata-Mestrov 1961, p. 75), et "Alle Gnathopodenglieder sind am hinteren Rand sägeförmig gezähnt ebenso die Palma des Propode»(B.denticulata - Mestrov 1961, 0p. 77).

Il faut souligner que dans son travail de 1936, Hertzog en présentant une nouvelle description de son espèce, donne pour B.albertimagni les caractéristiques trouvées par Mestrov pour B.denticulata: "...tranchant les propodes sous les dactyles en lamelle hyaline finement crénelée», ou "le bord interne du propode est finement et obliquement serrulé en scie...» (Hertzog 1936, p. 362). Plus loin, il ajoute: "Profil du bord interne (post. ou inf.) du basal et de l'ischial de gn. I et II serrulé et cilié en scie oblique» (Hertzog 1936, p. 364).

En tenant compte que dans sa bibliographie Mestrov n'indique pas le travail de Hertzog (1936), nous sommes tentés de supposer qu'il ne l'a pas connu. Ce fait, ainsi que l'évidente concordance entre les caractéristiques de B.albertimagni et B.denticulata, imposent la conclusion qu'elles représentent la même espèce.

7) «Gezähnte Gnathopodenglieder sind bei dieser Gattung bisher nicht bekannt» (Mestrov 1961, p. 78). 


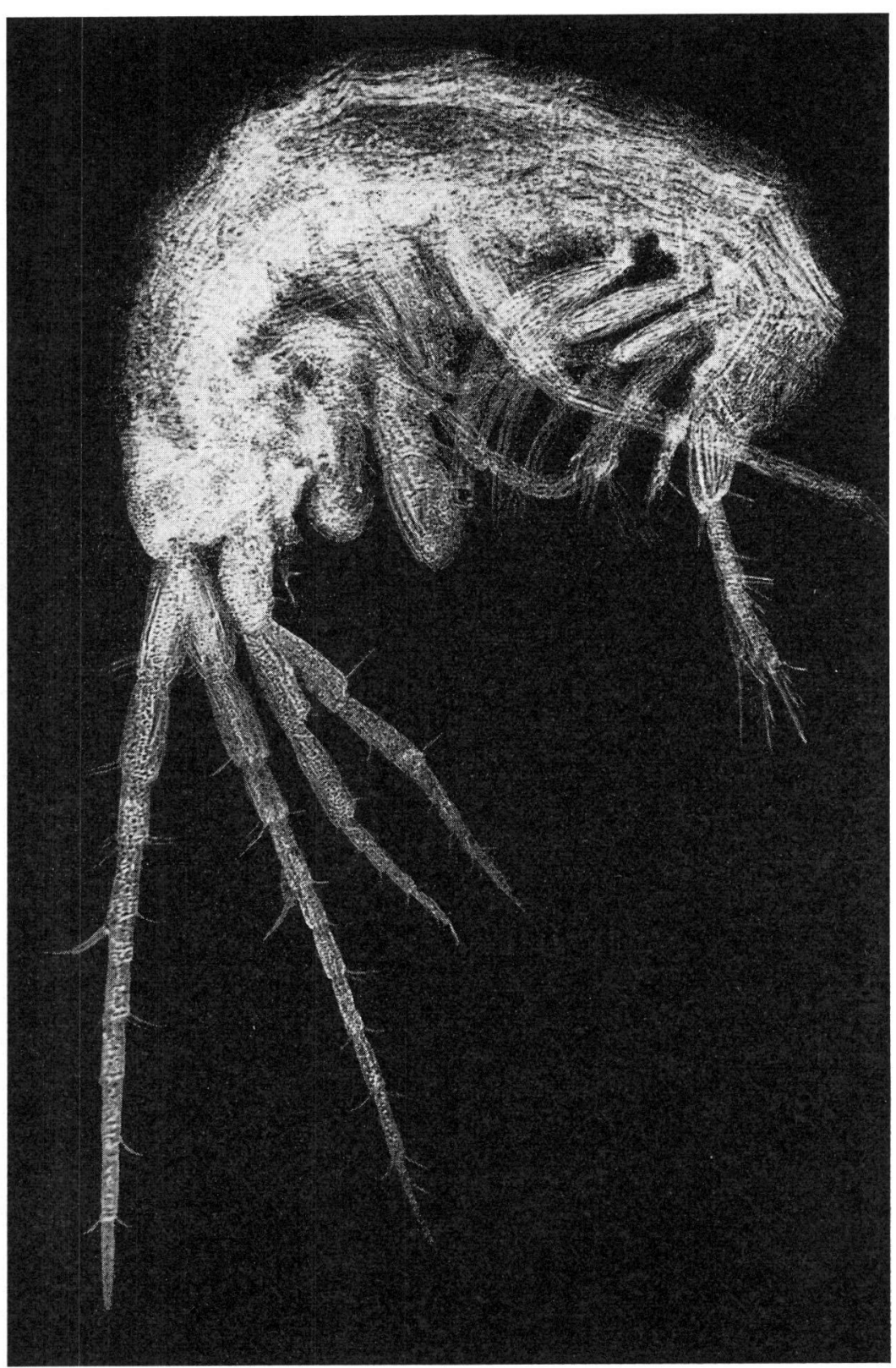

Fig. 1. Bogidiella albertimagni Hertzog. 


\section{RÉSUMÉ}

Ayant étudié quelques exemplaires de Bogidiella collectés de la nappe phréatique de la Vallée Cerna (Olténie) au moyen du procédé des sondages Karaman-Chappuis, les auteurs annoncent la présence de B.albertimagni Hertzog en Roumanie.

Après une sommaire description des exemplaires trouvés, on discute quelques problèmes concernant la valeur taxonomique de B. albertimagni Hertzog et B.skopljensis Karaman (déjà connue de Roumanie) et la validité de $B$.denticulata Mestrov décrite de Yougoslavie.

\section{SUMMARY}

Studying several Bogidiella individuals collected from the phreatic biotope of Cerna Valley (Oltenia region) using Karaman-Chappuis method, authors announce the presence of Bogidiella albertimagni Hertzog in Rumania.

After a description of the studied individuals, one talk about some problems concerning the taxonomic value of B.albertimagni and B.skopljensis Karaman (this second species being formerly known in Rumania) and the validity of B.denticulata Mestrov described from Yugoslavia.

\section{BIBLIOGRAPHIE}

Garauşu, S., Dobreanu, E., şi Manolache, C. (1959) - Crustacea-Amphipoda. Forme salmastre şi de apa dulce. Fauna R. P. R., IV, 4.

Dobreanu, E., şi Manolache, C. (1951) - Noi Amphipode de apa dulce pentru fauna R. P. R. Com. Acad. RPR, I, 11-12: 1056-1059.

Hertzog, L. (1933) - Bogidiella albertimagni sp. n., ein neuer Grundwasseramphipode aus der Rheinebene bei Straßburg. Zool. Anz., 102: $225-227$.

- (1935) - Amphipoden aus dem Grundwasser von Skoplje. Zool. Anz., 111: $50-52$.

- (1936) - Crustacés des biotopes hypogées de la Vallée du Rhin d'Alsace. Bull. Soc. Zool. France, 61: 356-372.

Husmann, S. (1956) - Untersuchungen über die Grundwasserfauna zwischen Harz und Weser. Arch. f. Hydrob., 52: 1-184.

Karaman, S. (1933) - Über zwei Amphipoden Balcanella und Jugocrangonyx, aus dem Grundwasser von Skoplje. Zool. Anz., 103, 1/2: 41-47.

- (1934) - Die unterirdischen Amphipoden Südserbiens. Srpska Kr. Akad. 135: 163-313.

- (1953) - Über subterrane Amphipoden und Isopoden des Karstes von Dubrovnik und seines Hinterlandes. Acta Musei Maced. Sci. Nat., I, 7: 137-167.

- (1959) - Über eine neue Art und Unterart der Gattung Bogidiella (Crust. Amphipoda) aus Jugoslawien. Acta Zool. Acad. Scient. Hungaricae, 4, 3-4: 339-348.

Mestrov, M. (1961) - Über neue Bogidiella-Arten (Crustacea-Amphipoda) aus unterirdischen Gewässern Kroatiens und Sloweniens. Zool. Anz., 167: $74-80$.

Ruffo, S. (1952) - Bogidiella neotropica n.sp. nuovo Anfipodo dell'Amazonia. Rivista Svizzera di Idrologia, 14, 1: 129-134. 
Ruffo, S. (1953a) - Anfipodi di acque interstiziali raccolti dal dr. C. Delamare Deboutteville in Francia, Spagna e Algeria. Vie et Milieu, 4, 3: 669-681.

- (1953b) - La stato attuale delle conoscenze sulla distribuzione geografica degli Anfipodi. Publ. du Premier Congrès Int. de Spéléologie, Paris, 3: 13-37.

- (1954) - Bogidiella chappuisi Ruffo nouvel amphipode phréatobie de la faune française. Arch. Zool. exp. et gén., 91, 1: 145-152.

- (1958) - Studi sui Crostacei Anfipodi LIII. Due nuove specie di Anfipodi delle acque sotterranee dell'Afghanistan, Mem. del Museo Civico di St. Nat., 6: 389-403.

- (1963) - Studi sui Crostacei Anfipodi LVII. Una nuova specie di Bogidiella (Crust. Amphipoda) della depressione del Mar Morto. Bull. research coun. Israel, $11 \mathrm{~B}: 4$.

Ruffo, S., et Delamare Deboutteville, Cl. (1952) - Deux nouveaux Amphipodes souterrains de France: Salentiella angelieri n. sp. et Bogidiella chappuisi n. sp. C. R. Séances de l'Acad. des Sciences. 234: 1636-1638.

Schellenberg, A. (1942) - Flohkrebse oder Amphipoda. Tierwelt Deutschlands, 40, Krebstiere oder Crustacea, 4: 252.

Shozmaker, C. R. (1959) - Three new cave Amphipods from the West Indies. J. of the Washington Acad. of. Sci., 49, 8: 273-283.

Siewing, R. (1953) - Bogidiella brasiliensis, ein neuer Amphipode aus dem Küstengrundwasser Brasiliens. Kieler Meeresforsch., 9, 2: 243-247. Spooner, G. M. (1959) - News Members of the British Marine Bottom Fauna. Nature, 183: 1695-1696.

- (1960) - The occurrence of Ingolfiella in the Eddystone shell gravel, with description of new species. J. mar. biol. Ass. U. K., 39: 319-329.

- (1961) - Bathynella and other interstitial Crustacea in Southern England. Nature, 190, 4770: 104-105.

Villalobos, A. (1960) - Un Anfipodo cavernicola nuevo de Mexico: Bogidiella tabascensis n.sp. An. Inst. Biol. Mex., 31: 317-334.

EXPLICATION DES PLANGHES 71 (1) - 75 (5)

\section{PLANGHE $71(1)$}

Bogidiella albertimagni Hertzog; aspect général.

\section{PLANCHE $72(2)$}

Bogidiella albertimagni Hertzog; A - antenne I; B - antenne II; C, D mandibule; $\mathrm{E}$ - maxille $\mathrm{I} ; \mathrm{F}$ - maxille II $; \mathrm{G}$ - maxillipède.

\section{PLANGHE $73(3)$}

Bogidiella albertimagni Hertzog; A - gnathopode I; B - gnathopode II; $\mathrm{C}$-péréiopode III ; D - péréiopode IV; $\mathrm{E}$-péréiopode V; F-péréiopode VI; $\mathrm{G}$ - péréiopode VII. 


\section{PLANCHE 74 (4)}

Bogidiella albertimagni Hertzog; A, B - flagelle accessoire des antennes du même individu; $\mathrm{C}$ - labium; D - articles III et IV du palpe du maxillipède; $\mathrm{E}$ - bord palmaire et dactyle du gnathopode II; G, H, I - telson.

\section{PLANCHE 75 (5)}

Bogidiella albertimagni Hertzog; A, B, C-pléopodes I, II, III; D - plaques épimérales; $\mathrm{E}, \mathrm{F}, \mathrm{G}$ - uropodes I, II, III; $\mathrm{H}$ - telson; 1-7 plaques coxales. 


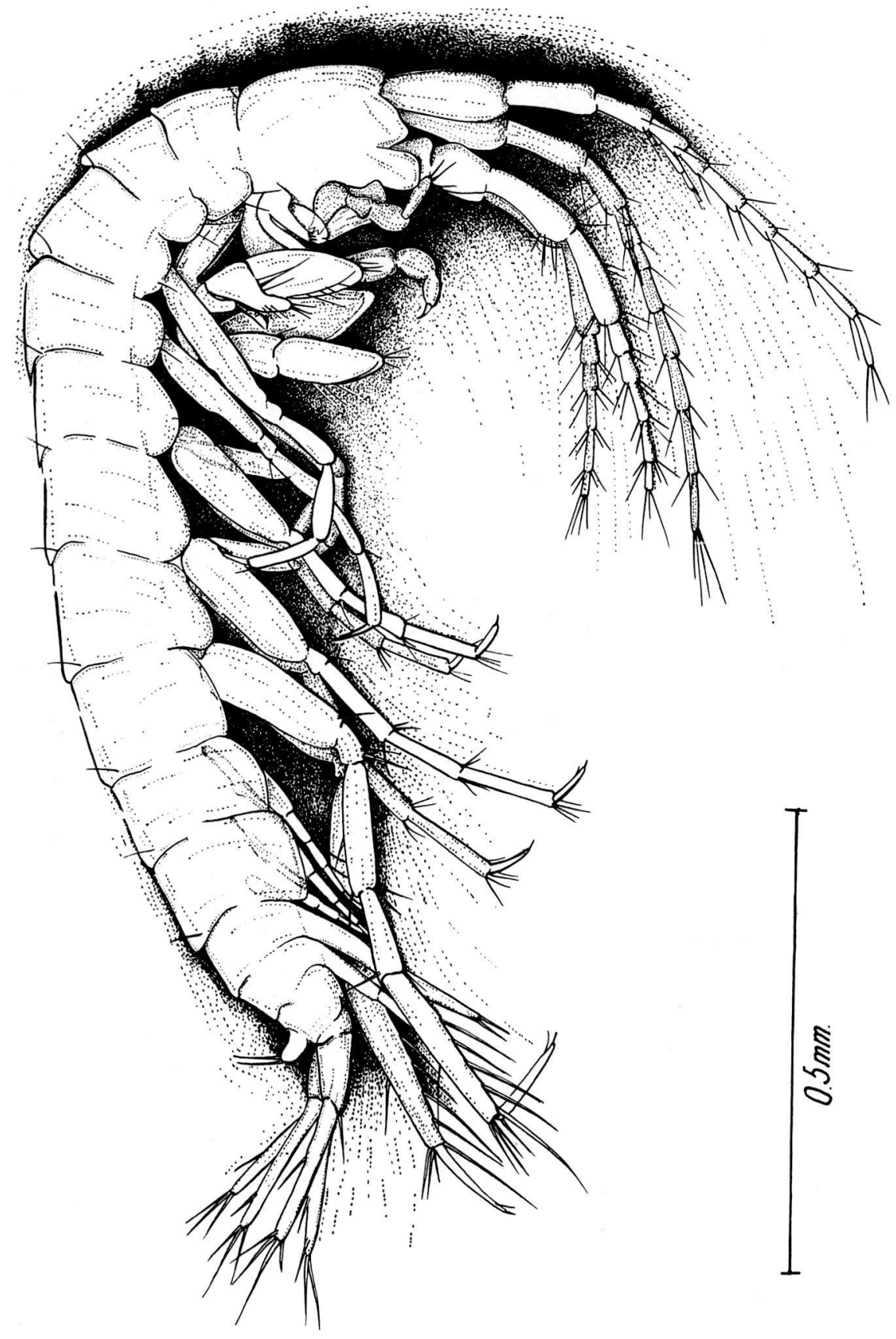




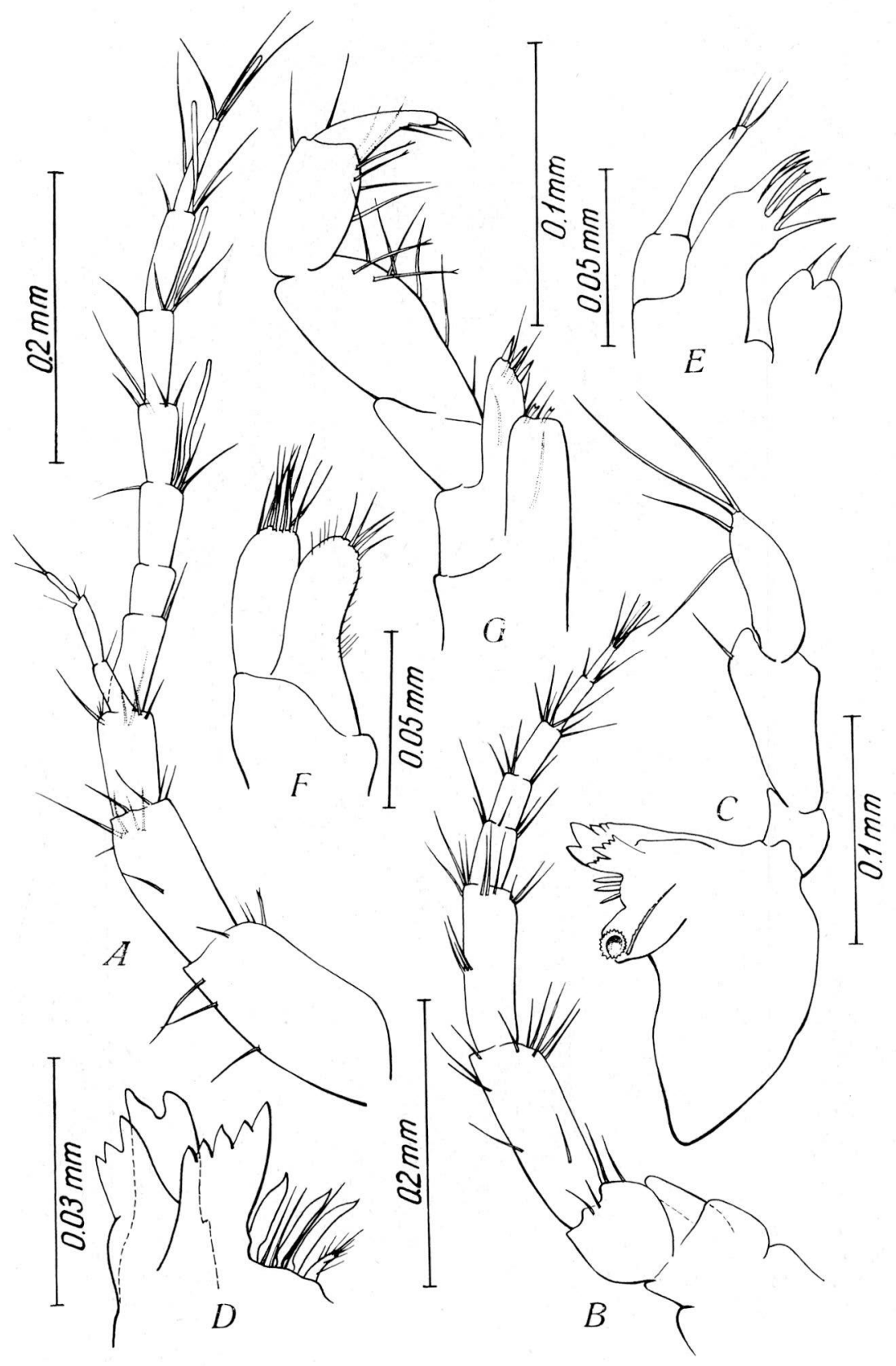




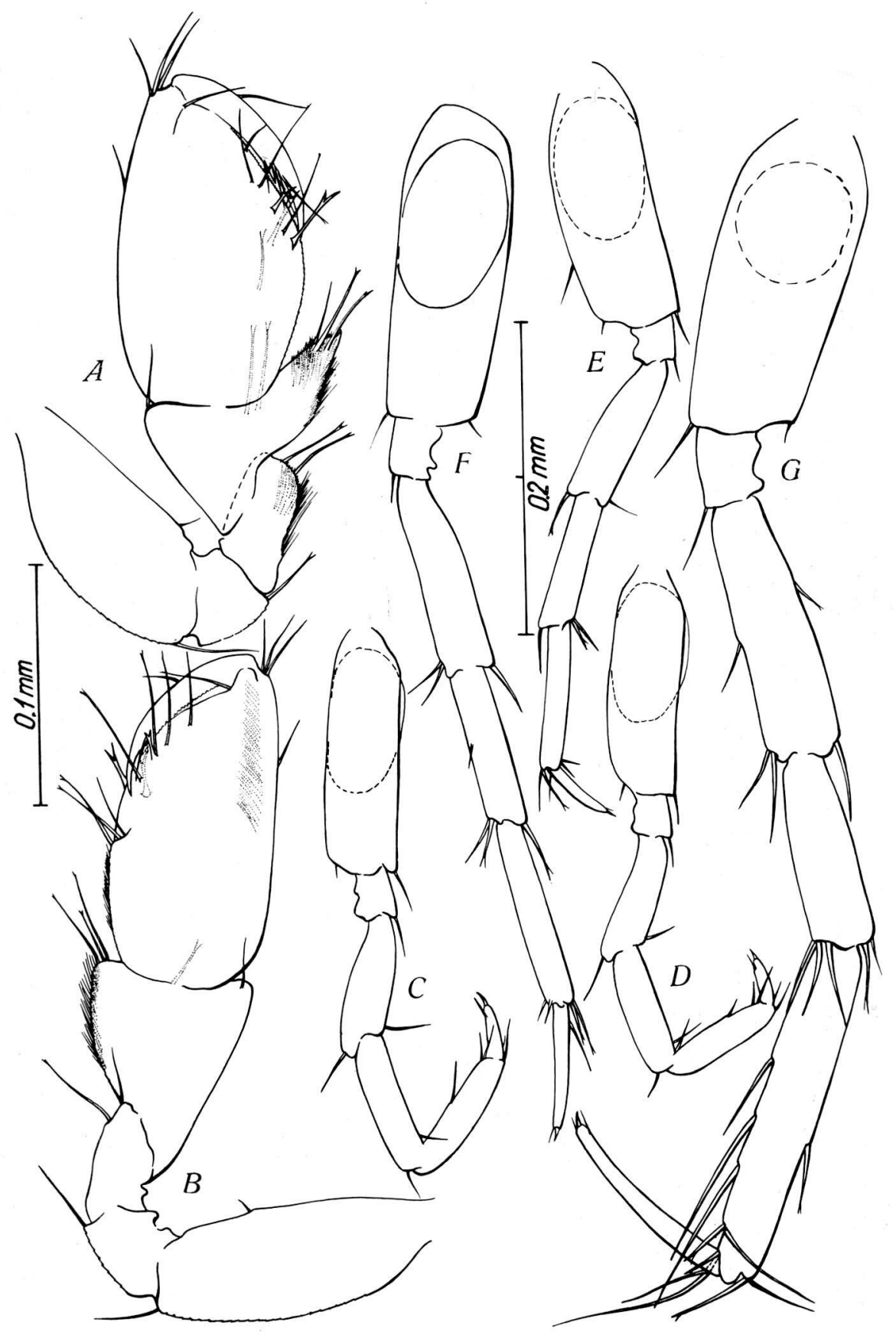




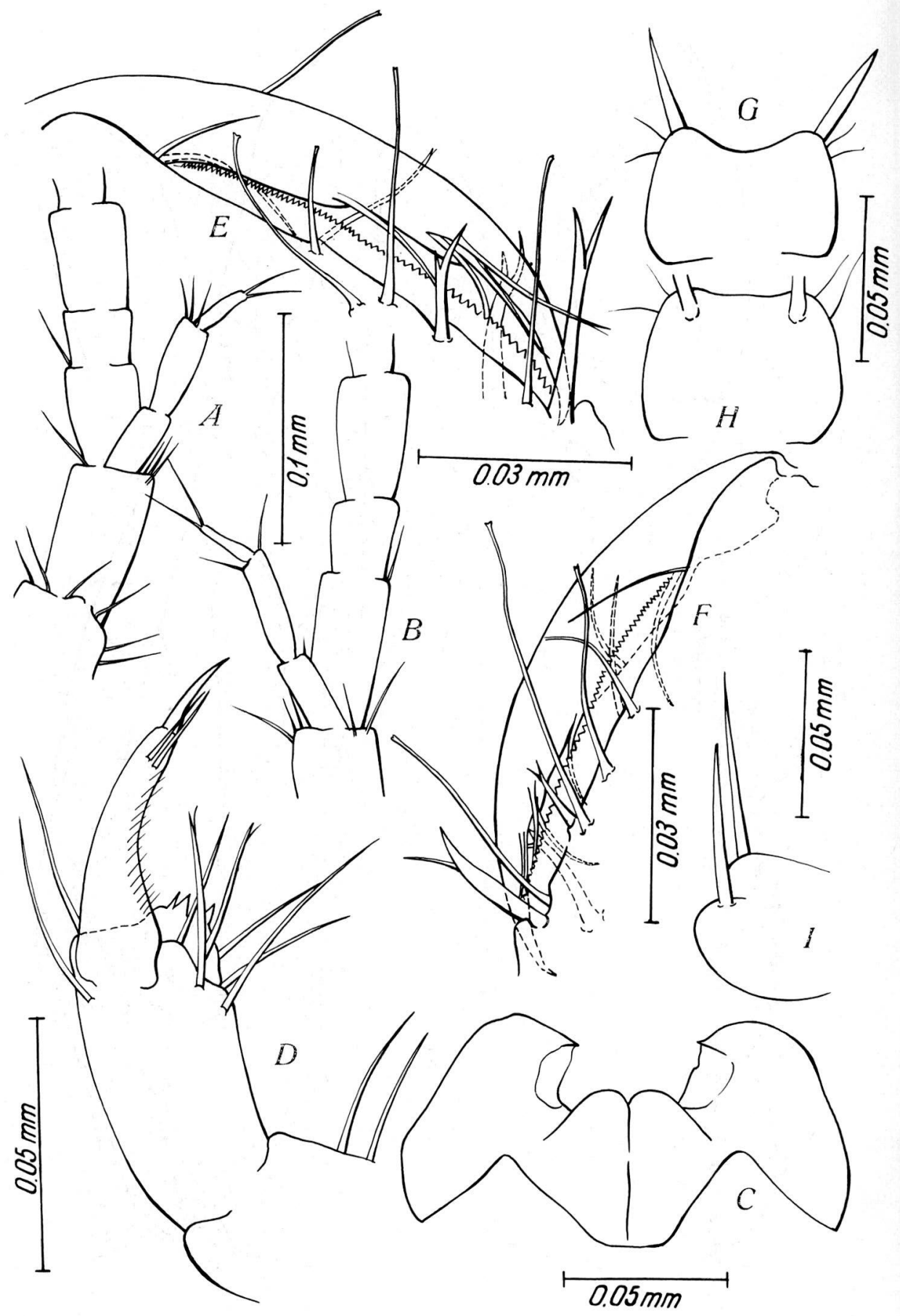




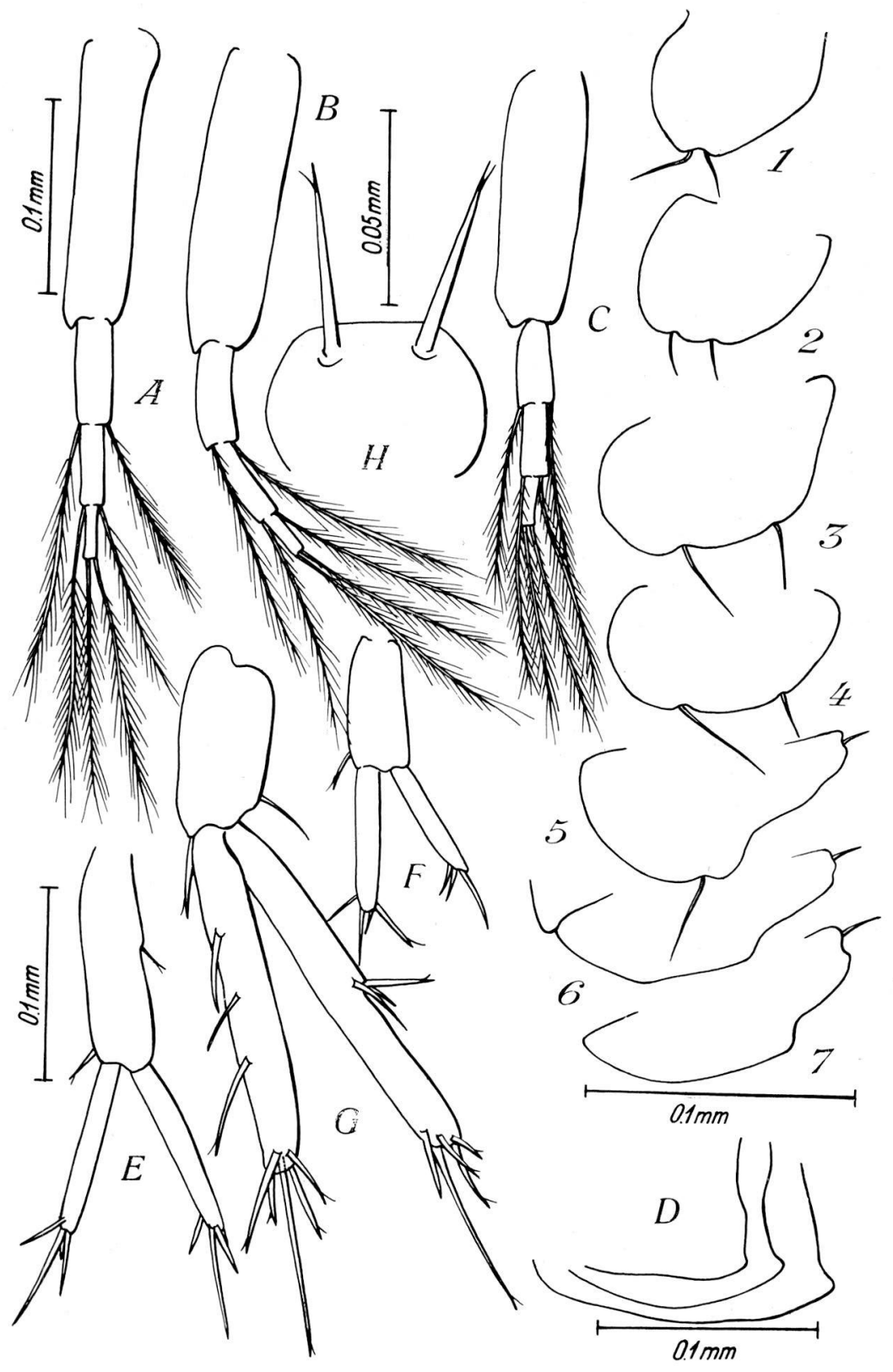

